\title{
Bivariate normal distribution for finding inliers in Hough space for a Time Projection Chamber
}

\author{
Amir Noori Shirazi ${ }^{1, a}$ and Ivor Fleck ${ }^{1, b}$ \\ ${ }^{1}$ Department Physik, Universität Siegen, Walter-Flex-Str.3, D57068, Siegen, Germany
}

\begin{abstract}
A Time Projection Chamber (TPC) is foreseen as the main tracking detector for the International Large Detector (ILD), one of the two detectors for the next candidate collider named International Linear Collider (ILC) [1].

GridPix, a combination of a micropattern gaseous detector with a pixelized readout, is one of the candidate readout systems for the TPC [2] [3]. One of the challenges in the track reconstruction is the large number of individual hits along a track (around 100 per $\mathrm{cm})$. Due to the small pixel size of $55 \times 55 \mu \mathrm{m}^{2}$, the distance between the individual ionization processes in the gas is larger than the size of the pixels. Consequently, the hits in the GridPix are not contiguous. This leads to the challenge of assigning the individual hits to the correct track. Hits within a given distance from a reconstructed track are called inliers. Consequently, finding inliers within the large number of hits and in the presence of noise is difficult for pattern recognition. This difficulty is increased by diffusion effects in the TPC.

One of the current algorithms which are utilized for track finding is the Hough transform. Using a bivariate normal distribution based on the covariance matrix calculated from the diffusion effect improves collecting inliers in the Hough space directly [5].
\end{abstract}

\section{Introduction}

The International Linear Collider (ILC) to be built in Japan, is one of the main candidate colliders for the future of high energy physics. The ILC has two main detectors: International Large Detector (ILD) and Silicon Detector (SiD). The main tracking detector for ILD is a Time Projection Chamber (TPC) with benefits such as low material budget and continuous tracking [1].

GridPix, one of the candidate readout systems for the TPC, is a combination of a micropattern gaseous detector with a TimePix chip readout system [2] [3]. The result of this combination is that there are many individual hits along a track (around 100 per $\mathrm{cm}$ ). The size of each pixel is $55 \times 55$ $\mu \mathrm{m}^{2}$ and in each chip, there are $256 \times 256$ pixels. Every 8 GridPix chips are aligned in a structure called Octoboard [2]. One of the main reasons to use GridPix is to reach the highest possible double track resolution for the ILD-TPC. A large number of hits makes it very challenging for the pattern recognition to assign a hit to the correct track. This difficulty is increased because of noise and diffusion effects in the TPC.

\footnotetext{
ae-mail: shirazi@hep.physik.uni-siegen.de

be-mail: fleck@hep.physik.uni-siegen.de
} 
The strategy that is used here is to find inliers for a small segment of a track, called tracklet, and then to connect all relevant tracklets to each other in order to have the full track. One of the advantages of this method is, that finding inliers locally is easier than using a global method for the full track. For this study, the unit of the Octoboard is for finding a segment of the track. The size of each Octoboard is $45 \times 65 \mathrm{~mm}^{2}$ and this allows for high momentum particles, to assume a straight line for each segment even with a magnetic field in the range of $4 \mathrm{~T}$. It is obvious that this assumption is not correct for low momentum particles, for instance in the range of a few $\mathrm{MeV}$.

Hough transform is one of the typical methods used in image processing, computer vision and also in high energy physics to find the parameters of a line or curve fitted on many points or hits in an image space based on a voting process. According to Richard Duda and Peter Hart, the normal equation of a straight line becomes [4]:

$$
\rho=x \cos (\phi)+y \sin (\phi)
$$

Where $\rho$ and $\phi$ are the normal parameters of the straight line. Based on equation (1) each hit generates a sinusoid shaped line in Hough space and the lines of all hits intersect in a bin that corresponds to a parameter vector. In order to find the track parameters, the bin with the maximum entries in Hough space is taken and this bin returns the candidate parameters of a candidate track [6]. As mentioned before, due to the diffusion effects in the TPC, the width of a track increases with the drift distance and for a fixed size of the bins in Hough space, some lines for inliers do not pass through the bin corresponding to the track parameters.

This paper explains how to use a bivariate normal distribution (BND) fit in Hough Space around the bin with the maximum entries in order to collect inliers in Hough space [5]. With this method, we are able to separate two tracks from each other and also to separate track hits from noise hits. In the next section we explain how to calculate BND fit parameters based on the diffusion coefficient.

\section{Diffusion and Covariance matrix}

After the primary ionization created by a charged particle along its trajectory inside a TPC, a charge cloud of electrons from ionization in a gas diffuses because of the thermal movement of the gas molecules. The diffusion effect increases with the drift distance and causes the charge cloud, after some time $t$ to show a Gaussian density distribution as follows [7] [8]

$$
n=\frac{1}{\sqrt{4 \pi \tilde{D_{L}} t}}\left(\frac{1}{\sqrt{4 \pi \tilde{D_{T}} t}}\right)^{2} \exp \left(-\frac{x^{2}+y^{2}}{4 \tilde{D_{T}} t}-\frac{\left(z-v_{d} t\right)^{2}}{4 \tilde{D}_{L} t}\right)
$$

Where $v_{d}$ is the drift velocity and $\tilde{D_{T}}$ and $\tilde{D_{L}}$ are transverse and longitudinal diffusion coefficient respectively. When applying an electric field for drifting, the diffusion in the $\mathrm{Z}$ direction (along the electric field) is different from the $\mathrm{X}$ and $\mathrm{Y}$ directions which are perpendicular to the electric field. For this reason, there are two diffusion coefficients, one for the $\mathrm{X}$ and $\mathrm{Y}$ directions $\left(\tilde{D_{T}}\right)$ and another one for the $\mathrm{Z}$ direction $\left(\tilde{D}_{L}\right)$. According to equation (2) there are two resolutions as follows:

$$
\sigma_{T}=\sqrt{2 \tilde{D_{T}} t} \text { and } \sigma_{L}=\sqrt{2 \tilde{D_{L}} t}
$$

Where $\sigma_{T}$ and $\sigma_{L}$ are transverse and longitudinal resolution respectively. In order to eliminate the dependency on the time, diffusion constants are defined as $D_{T}=\sqrt{\frac{2 \tilde{D_{T}}}{v_{d}}}$ and $D_{L}=\sqrt{\frac{2 \tilde{D_{L}}}{v_{d}}}$ for both diffusion coefficients. The diffusion constants have a dependency on the gas mixture used in the TPC. 
With implementing a magnetic field parallel to the electric field, the trajectory of the electrons has a helix shape and it causes the transverse diffusion constant to be different from that without magnetic field. In addition to the diffusion the resolutions also depend on the readout system. The transverse resolution has a contribution $\sigma_{0}=0.055 \mathrm{~mm} / \sqrt{12}$ from the pitch of the pixels and the longitudinal resolution a contribution due to the frequency $\left(f_{c}\right)$ of the readout system. In our case $f_{c}=40 \mathrm{MHz}$ [9]. In this case, the transverse and longitudinal resolution have the form:

$$
\begin{aligned}
\sigma_{T} & =\sqrt{\sigma_{0}^{2}+Z_{d r i f t} D_{T}^{2}} \\
\sigma_{L} & =\sqrt{\frac{1}{6}\left(\frac{v_{d}}{f_{c}}\right)^{2}+Z_{d r i f t} D_{L}^{2}}
\end{aligned}
$$

Which shows the resolution of a track in dependence on the drift length, the gas mixture and the magnetic field [8] [9].

Generally, for GridPix as the readout system, because of the small size of the pixels, only a single electron is detected in one readout channel. Therefore there is no correlation between $\mathrm{X}$ and $\mathrm{Y}$ coordinates. Consequently, the covariance matrix is a $3 \times 3$ diagonal matrix based on the transverse and longitudinal diffusion.

$$
\operatorname{cov}=\left[\begin{array}{ccc}
\sigma_{T}^{2} & 0 & 0 \\
0 & \sigma_{T}^{2} & 0 \\
0 & 0 & \sigma_{L}^{2}
\end{array}\right]
$$

For the ideal case, when a fixed size of the binning is used for Hough space, and the width of the charge cloud is small, the lines of all hits pass through one bin. This bin then corresponds to track parameters in Hough space. But when the diffusion is large, then for the same binning, some lines could not pass through the bin with maximum entries. For this reason, in order to find inliers directly inside the Hough space, we calculate the resolution of the track parameters in this bin. The following section explains how it is done. Figure 1 is an example for the image and Hough space for a track.
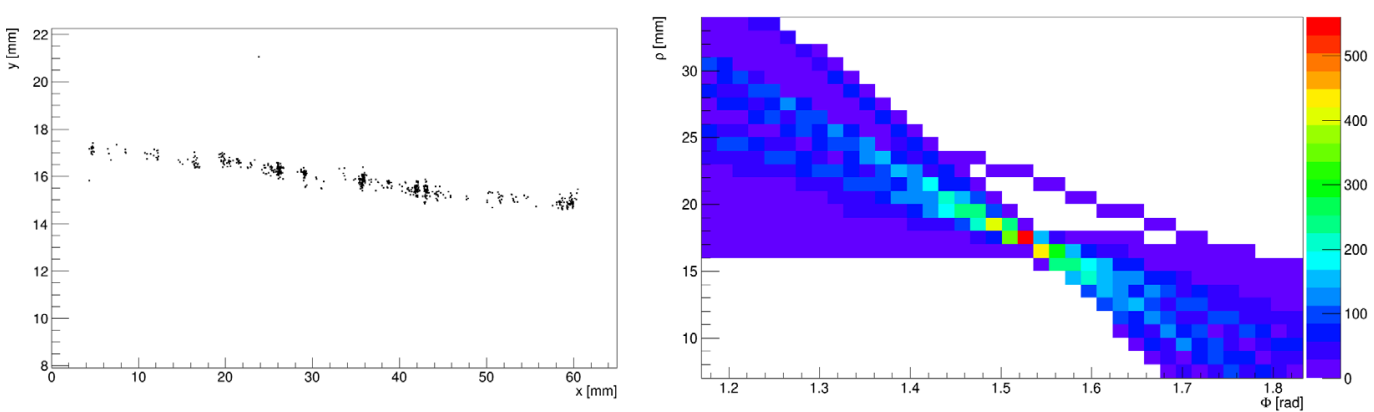

Figure 1: The left figure shows an example of a track in the image space (XY-Plane) and the right figure shows the Hough space for the same hits. Because of the diffusion, some lines for hits do not pass through the bin with maximum entries. 


\subsection{Covariance matrix in the XY-Plane}

As mentioned before, we use the normal equation for Hough transform. For the first step we calculate the track's parameters only in the XY-Plane. In this case, the covariance matrix for image space (or for hits space) is

$$
V_{i j}=\left[\begin{array}{cc}
\sigma_{T}^{2} & 0 \\
0 & \sigma_{T}^{2}
\end{array}\right]
$$

The next step is to calculate the covariance matrix in Hough space. For this calculation, we use first-order error propagation:

$$
\left[\begin{array}{cc}
\sigma_{\rho}^{2} & \sigma_{\rho \phi} \\
\sigma_{\rho \phi} & \sigma_{\phi}^{2}
\end{array}\right]=J\left[\begin{array}{cc}
\sigma_{T}^{2} & 0 \\
0 & \sigma_{T}^{2}
\end{array}\right] J^{T}
$$

With $\sigma_{\rho \phi}, \sigma_{\rho}$ and $\sigma_{\phi}$ are covariance and variance of $\rho$ and $\phi$ in Hough Space, respectively. In addition, $J$ is the Jacobian matrix of equation (1) [10].

$$
J=\left[\begin{array}{ll}
\frac{\partial \rho}{\partial x} & \frac{\partial \rho}{\partial y} \\
\frac{\partial \phi}{\partial x} & \frac{\partial \phi}{\partial y}
\end{array}\right]
$$

\subsection{Covariance matrix in the SZ-Plane}

In order to separate two tracks or better say to find inliers in the $\mathrm{Z}$ direction, the Hough transform is also implemented in the SZ-Plane. S is defined as the arc length in the XY-Plane, and in the case of $\mathrm{B}=0$ (or straight tracks), is the shortest distance between a hit and the point of closest approach (pca). The pca is the closest point on a track to the reference point (here: the origin of the coordinate system) [11].

$$
S=\sqrt{\left(x_{h i t}-x_{p c a}\right)^{2}+\left(y_{h i t}-y_{p c a}\right)^{2}}
$$

For calculating $\sigma_{S}$ first order error propagation is used based on equation (9)

$$
\sigma_{S}^{2}=\left(\frac{\partial S}{\partial x}\right)^{2} \sigma_{T}^{2}+\left(\frac{\partial S}{\partial y}\right)^{2} \sigma_{T}^{2}
$$

In the SZ-Plane, the covariance matrix is defined as:

$$
V_{i j}=\left[\begin{array}{cc}
\sigma_{S}^{2} & 0 \\
0 & \sigma_{L}^{2}
\end{array}\right]
$$

For the Hough transform in the SZ-Plane we use:

$$
\rho_{z}=S \cos (\theta)+z \sin (\theta)
$$

And therefore we have

$$
\left[\begin{array}{cc}
\sigma_{\rho_{z}}^{2} & \sigma_{\rho_{z} \theta} \\
\sigma_{\rho_{z} \theta} & \sigma_{\theta}^{2}
\end{array}\right]=J\left[\begin{array}{cc}
\sigma_{S}^{2} & 0 \\
0 & \sigma_{L}^{2}
\end{array}\right] J^{T}
$$

With $J$ is, as before, the Jacobian matrix [10]:

$$
J=\left[\begin{array}{ll}
\frac{\partial \rho_{z}}{\partial S} & \frac{\partial \rho_{z}}{\partial z} \\
\frac{\partial \theta}{\partial S} & \frac{\partial \theta}{\partial z}
\end{array}\right]
$$




\section{Bivariate normal distribution (BND)}

In order to find inliers and outliers in Hough space, bivariate normal distribution is utilized around the bin with maximum entries in Hough space. Generally, a bivariate normal distribution shows the distribution of two parameters which could have a correlation. In Hough space, two parameters (for 2D) $\phi$ and $\rho$ have a correlation. The general equation for the bivariate normal distribution is:

$$
G(\phi, \rho)=\frac{1}{2 \pi \sigma_{\rho} \sigma_{\phi} \sqrt{1-r^{2}}} \exp \left(-\frac{c}{2\left(1-r^{2}\right)}\right)
$$

where

$$
c \equiv \frac{\left(\phi-\mu_{\phi}\right)^{2}}{\sigma_{\phi}^{2}}-\frac{2 r\left(\phi-\mu_{\phi}\right)\left(\rho-\mu_{\rho}\right)}{\sigma_{\phi} \sigma_{\rho}}+\frac{\left(\rho-\mu_{\rho}\right)^{2}}{\sigma_{\rho}^{2}}
$$

and $\mu_{\phi}$ and $\mu_{\rho}$ are the mean value for $\phi$ and $\rho$ respectively and $\mathrm{r}$ is the correlation

$$
r \equiv \frac{\sigma_{\rho \phi}}{\sigma_{\rho} \sigma_{\phi}}
$$

The Bivariate normal distribution has an elliptical shape. In case there is a correlation between the two parameters, this ellipse has a tilt to the x-axis otherwise it would be a vertical or horizontal ellipse (depends on the minor and major semi-axis). In Eq (15), the term before the exponential part is the normalization factor [13].

After fitting the BND in Hough space around the bin with maximum entries using ROOT [12], there is an iso-contour of equation (15) which has an elliptical shape and then one can find the semiminor and semi-major axis of the ellipse corresponding to the $1 / \mathrm{e}$ of the peak of the BND fit. This ellipse corresponds to the one sigma interval of the fit. A hit that its line in Hough space passes through this ellipse is called an inlier. The important point of this method is the size of the ellipse is changeable which means the number of inliers can be changed. Figure 2 shows the BND fit in Hough space (a) and the ellipse corresponding to the $1 \sigma$ interval of the (b).

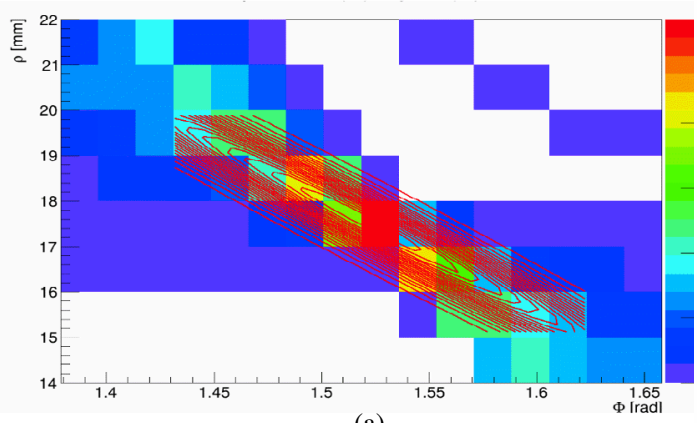

(a)

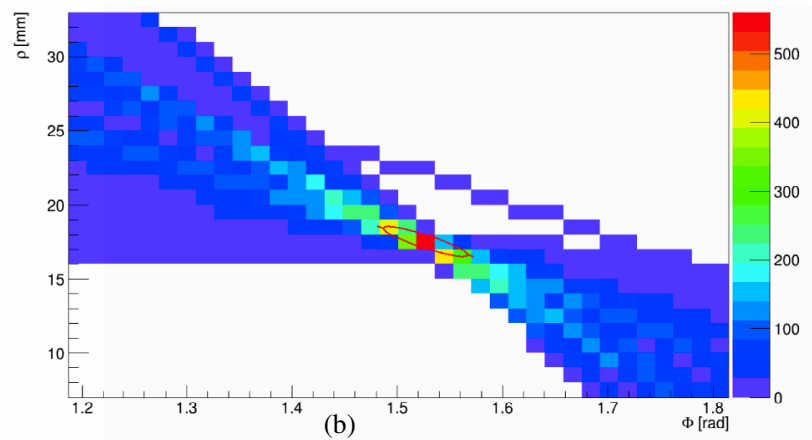

(b)

Figure 2: (a) Iso-countor of the BND fit and (b) the ellipse corresponding to 1/e of the peak value.

From equation(15) and (16) we have

$$
1=\frac{\left(\phi-\mu_{\phi}\right)^{2}}{q \sigma_{\phi}^{2}}+\frac{\left(\rho-\mu_{\rho}\right)^{2}}{q \sigma_{\rho}^{2}}-\frac{2 r\left(\phi-\mu_{\phi}\right)\left(\rho-\mu_{\rho}\right)}{q \sigma_{\phi} \sigma_{\rho}}
$$


and also generally the equation of a tilted ellipse is

$$
1=\left(\frac{\cos ^{2}(\alpha)}{a^{2}}+\frac{\sin ^{2}(\alpha)}{b^{2}}\right) \phi^{2}-2 \cos (\alpha) \sin (\alpha)\left(\frac{1}{a^{2}}-\frac{1}{b^{2}}\right) \phi \rho+\left(\frac{\sin ^{2}(\alpha)}{a^{2}}+\frac{\cos ^{2}(\alpha)}{b^{2}}\right) \rho^{2}
$$

therefore we have semi-major and semi minor of the ellipse as follow:

$$
\begin{aligned}
& a^{2}=\frac{q \sigma_{\phi}^{2} \sigma_{\rho}^{2} \cos (2 \alpha)}{\sigma_{\rho}^{2} \cos ^{2}(\alpha)-\sigma_{\phi}^{2} \sin ^{2}(\alpha)} \\
& b^{2}=\frac{-q \sigma_{\phi}^{2} \sigma_{\rho}^{2} \cos (2 \alpha)}{\sigma_{\rho}^{2} \sin ^{2}(\alpha)-\sigma_{\phi}^{2} \cos ^{2}(\alpha)}
\end{aligned}
$$

After finding the two ellipses for both, the XY-plane and the SZ-plane, we iterate over all hits and those having a line that passes through both ellipses are collected as inliers. Thereafter, a Karimäki Circle fit and a straight line fit are used for having pre-estimations of the parameters of the tracklet which later will be used for the merging process [14] [15].

\section{Result}

As mentioned before, one of the objectives of our study is to reach the highest possible double track resolution. As the first step, we simulated two electrons with an energy of $6 \mathrm{GeV}$ to pass through the TPC parallel to the X-axis. All simulations were done for the Large Prototype Time Projection Chamber (LP-TPC) at DESY with a 1T magnetic field. These simulations were done for two tracks with the same $\mathrm{Z}$ position and varying distances in $\mathrm{Y}(1-8 \mathrm{~mm}$ ) (figure 3 ) and then for the same condition but this time for the variation of the distance between $1 \mathrm{~mm}$ to $10 \mathrm{~mm}$ in the $\mathrm{Z}$ direction and the same $\mathrm{Y}$ offset (figure 4). All simulations were done near the full drift length of $600 \mathrm{~mm}$ in order to have the maximum diffusion effect. For each step 1000 events were generated, with up to 500 additional random noise hits for each event.

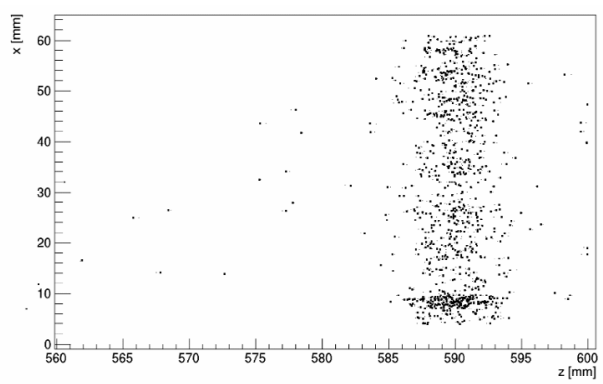

(a)

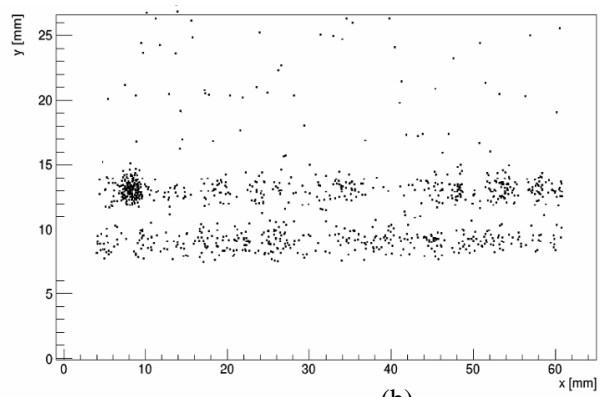

(b)

Figure 3: Example for two tracks with the same $\mathrm{Z}$ offset and $\Delta Y=4 \mathrm{~mm}$.(a) The two tracks in the XZ-Plane, and (b) the same two tracks in the XY-Plane. 


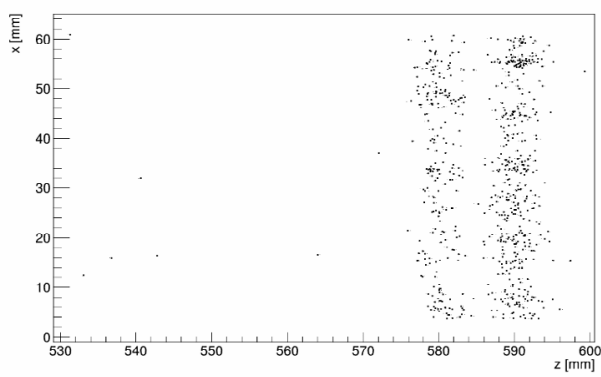

(a)

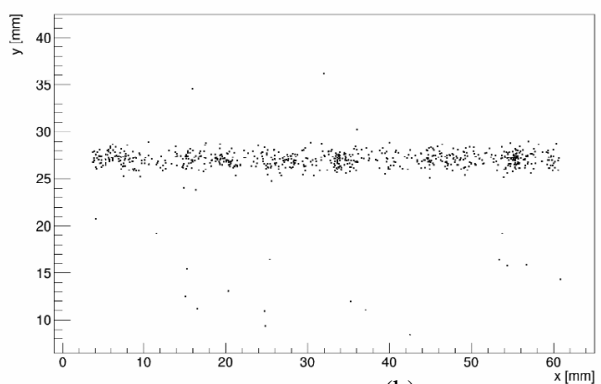

(b)

Figure 4: Example for two tracks with the same $\mathrm{Y}$ offset and $\Delta Z=4 \mathrm{~mm}$.(a) The two tracks in the XZ-Plane, and (b) the same two tracks in the XY-Plane.

The results of the reconstruction for different track distances are shown in figure 5. To calculate the track efficiency, a correct track is associated with its true Monte Carlo particle if it has more than some percentage (e.g. $60 \%$ or $70 \%$ ) of that MC particle's hits otherwise it is identified as a ghost track. In the case that a reconstructed track has sufficient hits for both MC particles, it is assigned to only one of them.

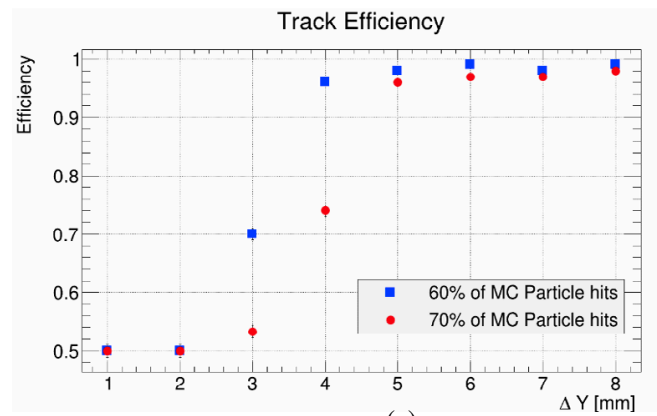

(a)

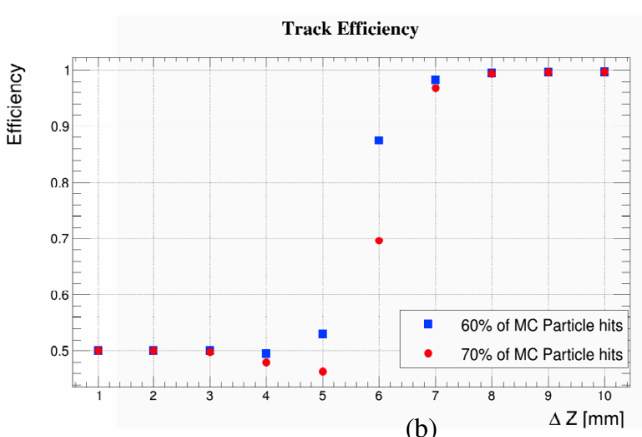

(b)

Figure 5: Track efficiency for tracks having more than $60 \%$ (blue points) and $70 \%$ (red points) of the true MC particle's hits.(a) Track efficiency as a function of $\Delta Y$. (b) Track efficiency as a function of $\Delta Z$.

For these simulations the transverse resolution $\left(\sigma_{T}\right)$ was about $0.7 \mathrm{~mm}$ and the longitudinal resolution $\left(\sigma_{L}\right)$ was approximately $2.0 \mathrm{~mm}$. According to the plot for $\Delta Y$ simulation, for correct tracks with more than $60 \%$ of MC particle's hits, we are able to separate double tracks with $4 \mathrm{~mm}$ distance from each other for more than $90 \%$ of the simulated tracks. However, the efficiency of this simulation when we consider correct tracks with $70 \%$ of correct MC particle hits decreases to around $75 \%$. For distance of $2 \mathrm{~mm}$ or less the two tracks cannot be separated any more and only one correct track can be reconstructed. This results in a track efficiency of $50 \%$. We can see the same behaviour for the $\Delta Z$ simulation plot for $6 \mathrm{~mm}$ distance between two tracks. 


\section{Conclusion}

In summary, we used a bivariate normal distribution fit in Hough space based on the covariance matrix calculated according to the transverse $\left(\sigma_{T}\right)$ and longitudinal $\left(\sigma_{L}\right)$ resolution in order to collect inliers directly in Hough space. The first study of this algorithm shows this method is promising. In the case that a correct track is associated with its true MC particle on condition that it has more than $60 \%$ of MC particle's hits, the simulation of two tracks for near the full drift length $(600 \mathrm{~mm})$ for LP-TPC shows that we can reach to more than $90 \%$ track efficiency when the distance of two tracks is $4 \mathrm{~mm}$ in the $\mathrm{Y}$ direction and have the same $\mathrm{Z}$ offset. This result is also true for the case that two tracks separated from each other with $6 \mathrm{~mm}$ distance in the $\mathrm{Z}$ direction and the same $\mathrm{Y}$ offset. The transverse resolution for this analysis and based on the equation (4) is about $0.7 \mathrm{~mm}$ and the longitudinal resolution is $1.8 \mathrm{~mm}$.

According to the LC-TPC characteristics, the double track resolution in the $r \phi$ plane should be around $2 \mathrm{~mm}$ and in the $r z$ plane should be almost $6 \mathrm{~mm}$ for the full drift length $(2.35 \mathrm{~m})$ of the TPC with a $3.5 \mathrm{~T}$ magnetic field [1]. In such a case, with the same electric field implemented in our simulation, the transverse resolution is about $0.4 \mathrm{~mm}$ and the longitudinal resolution is around $3.6 \mathrm{~mm}$ for the full drift length of the LC-TPC. As a conclusion, with extrapolation of our results to the LC-TPC conditions, we expect that this method will be able to achieve the double track resolution required for the LC-TPC.

In addition, comparing the longitudinal resolution required for the LC-TPC with that in this simulation shows that to reach the double track resolution in the $r z$ plane could be an issue, however, more studies and optimization of this method are ongoing. Different parameters are tuned to find any possibility of having better results and to improve this algorithm. The next step in finalizing the pattern recognition for a pixel readout TPC, will be to develop an algorithm for correctly merging the tracklets found with the method described here. 


\section{References}

[1] The International Linear Collider Technical Design Report - Volume 4: Detectors, 2013. CERNATS-2013-037, DESY 13-062, ILC-REPORT-2013-040.

[2] Michael Lupberger. The Pixel-TPC: A feasibility study. PhD thesis in Physik Bonn University, Germany, Bonn, August, 2015.

[3] X. Llopart et al. Timepix, a 65k programmable pixel readout chip for arrival time, energy and/or photon counting measurements., Nuclear Instruments and Methods in Physics Research Section A: Accelerators, Spectrometers, Detectors and Associated Equipment 581.1 (2007) 485-494, doi: 10.1016/j.nima.2007.08.079 (cit. on pp. 25, 30, 34, 112).

[4] Richard O.Duda and Peter E .Hart. Use of the Hough Transformation To Detect Lines and Curves in Pictures. Standford Research Institute, Menlo Park, California,1972.

[5] Leandro A.F.Fernandes, Manuel M .Oliveira. Real -time line detection through an improved Hough transform voting scheme. Elsevier,Pattern Recognition 41 (2008) 299 - 314.

[6] Richard O.Duda and Peter E .Hart. Use of the Hough Transformation To Detect Lines and Curves in Pictures. Standford Research Institute, Menlo Park, California,1972.

[7] W. Blum and L. Rolandi. Particle Detection with Drift Chambers. Springer, Berlin, 1993.

[8] Christoph Brezina. A GEM Based Time Projection Chamber with Pixel Readout. zur Erlangung des Doktorgrades (Dr. rer. nat.) der Mathematisch-Naturwissenschaftlichen Fakultät, der Rheinischen Friedrich-Wilhelms-Universität Bonn, 2013

[9] Martin Rogowski. Implementation of a track finding algorithm for an InGrid based detector. Masterarbeit in Physik Bonn University, Germany, Bonn, November, 2014.

[10] Kai Oliver Arras. An Introduction To Error Propagation: Derivation, Meaning and Examples of Equation $C_{Y}=F_{X} C_{X} F_{X}^{T}$. Technical Report No EPFL-ASL-TR-98-01 R3 of the Autonomous Systems Lab, Institute of Robotic Systems, Swiss Federal Institute of Technology Lausanne (EPFL),September 1998.

[11] Isa Heinze. Development of a Hough Transformation Track Finder for Time Projection Chambers. zur Erlangung des Doktorgrades, des Fachbereichs Physik, der Universität Hamburg, Hamburg, 2013.

[12] Rene Brun and Fons Rademakers. Root - an object oriented data analysis framework. Nucl. Instrum. Meth. A, 389:81-86, 1997.

[13] Glen Cowan. Statistical Data Analysis. Oxford University Press Inc., New York, 1998.

[14] Thomas Krämer. Track parameters in LCIO. 2006. LC-DET-2006-004.

[15] V. Karimäki. Effective circle fitting for particle trajectories. Nuclear Instruments and Methods in Physics Research Section A: Accelerators, Spectrometers, Detectors and Associated Equipment, 305:187-191, 1991. 